\title{
Potential utility of FLAIR in MRI-negative Cushing's disease
}

\author{
Grégoire P. Chatain, MD, MSc, ${ }^{1,4}$ Nicholas Patronas, MD, ${ }^{2}$ James G. Smirniotopoulos, MD, ${ }^{3}$ \\ Martin Piazza, MD, ${ }^{4}$ Sarah Benzo, RN, MPH, ${ }^{4}$ Abhik Ray-Chaudhury, MD, ${ }^{4}$ \\ Susmeeta Sharma, MD, ${ }^{5}$ Maya Lodish, MD, ${ }^{6}$ Lynnette Nieman, MD, ${ }^{7}$ \\ Constantine A. Stratakis, MD, PhD, ${ }^{6}$ and Prashant Chittiboina, MD, MPH ${ }^{1,4}$
}

\begin{abstract}
${ }^{1}$ Neurosurgery Unit for Pituitary and Inheritable Diseases, and ${ }^{4}$ Surgical Neurology Branch, National Institute of Neurological Disorders and Stroke; ${ }^{2}$ Diagnostic Radiology, Warren Grant Magnuson Clinical Center, National Institutes of Health; ${ }^{6}$ Section on Endocrinology and Genetics, Pediatric Endocrinology Inter-Institute Training Program, ${ }^{7}$ Eunice Kennedy Shriver National Institute of Child Health and Human Development, Bethesda, Maryland; ${ }^{2}$ Department of Radiology, George Washington University, Washington, DC; and ${ }^{5}$ Pituitary Endocrinology Section, MedStar Washington Hospital Center, Washington, DC
\end{abstract}

OBJECTIVE Accurate presurgical localization of microadenomas in Cushing's disease (CD) leads to improved remission rates and decreased adverse events. Volumetric gradient recalled echo (3D-GRE) MRI detects pituitary microadenomas in $C D$ in up to $50 \%-80 \%$ cases as a focus of hypointensity due to delayed contrast wash-in. The authors have previously reported that postcontrast FLAIR imaging may be useful in detecting otherwise MRI-negative pituitary microadenomas as foci of hyperintensity. This reflects theoretically complementary imaging of microadenomas due to delayed contrast washout. The authors report on the diagnostic accuracy and clinical utility of FLAIR imaging in the detection of microadenomas in patients with $C D$.

METHODS The authors prospectively analyzed imaging findings in 23 patients (24 tumors) with biochemically proven $\mathrm{CD}$ who underwent transsphenoidal surgery for $\mathrm{CD}$. Preoperatively, the patients underwent pituitary MRI with postcontrast FLAIR and postcontrast 3D-GRE sequences.

RESULTS Postcontrast FLAIR hyperintensity was detected in macroadenomas, and in 3D-GRE-positive or -negative microadenomas. Overall, 3D-GRE was superior in detecting surgically and histopathologically confirmed, location-concordant microadenomas. Of 24 pituitary adenomas, 18 (75\%; sensitivity $82 \%$, positive predictive value $95 \%$ ) were found on $3 \mathrm{D}-\mathrm{GRE}$, and 13 (50\% [ 1 was false positive]; sensitivity $55 \%$, positive predictive value $92 \%$ ) were correctly identified on FLAIR. The stand-alone specificity of 3D-GRE and FLAIR was similar (50\%). These results confirm the superiority of 3D-GRE as a stand-alone imaging modality. The authors then tested the utility of FLAIR as a complementary tool to 3D-GRE imaging. All 5 patients with negative 3D-GRE MRI displayed a distinct focus of FLAIR enhancement. Four of those 5 cases $(80 \%)$ had location-concordant positive histopathological results and achieved postsurgical biochemical remission. The remaining patient was not cured, because resection did not include the region of FLAIR hyperintensity.

CONCLUSIONS This study suggests that delayed microadenoma contrast washout may be detected as FLAIR hyperintensity in otherwise MRI-negative CD cases. The authors propose adding postcontrast FLAIR sequences to complement 3D-GRE for surgical planning in patients with $C D$.

Clinical trial registration no.: NIH protocol 03-N-0164, NCT00060541 (clinicaltrials.gov)

https://thejns.org/doi/abs/10.3171/2017.4.JNS17234

KEY WORDS FLAIR; fluid attenuated inversion recovery; 3D-GRE; volumetric gradient recalled echo; pituitary adenoma; hypercortisolism; Cushing's disease; transsphenoidal surgery; MRI; contrast; washout; pituitary surgery

ABBREVIATIONS $\mathrm{ACTH}=$ adrenocorticotropic hormone; $\mathrm{CD}=$ Cushing's disease; $\mathrm{CRH}=$ corticotropin-releasing hormone; $\mathrm{HDDST}=$ high-dose dexamethasone suppression test; IPSS = inferior petrosal sinus sampling; NIH = National Institutes of Health; NINDS = National Institute of Neurological Disorders and Stroke; PPV = positive predictive value; TSS = transsphenoidal surgery; UFC = urinary free cortisol; 3D-GRE = volumetric gradient recalled echo.

SUBMITTED January 26, 2017. ACCEPTED April 4, 2017.

INCLUDE WHEN CITING Published online October 13, 2017; DOI: 10.3171/2017.4.JNS17234. 
A CCURATE preoperative localization of microadenomas in Cushing's disease (CD) leads to improved remission rates and decreased adverse events. ${ }^{3,32}$ Modern MRI modalities including dynamic or volumetric sequences can detect corticotrophic adenomas in 50\%$80 \%$ of cases of CD. ${ }^{5,6,10,18,23,26}$ Typically, the rest undergo preoperative inferior petrosal sinus sampling (IPSS) to confirm a pituitary source of adrenocorticotropic hormone (ACTH)-dependent hypercortisolemia in patients with negative MRI findings. ${ }^{8,35}$ The IPSS, however, does not improve tumor localization within the pituitary parenchyma for surgical planning ${ }^{49}$ and it remains a poor guide for hemihypophysectomy. ${ }^{15,25}$

In postcontrast images, microadenomas in patients with $\mathrm{CD}$ are visualized as foci of hypointensity due to delayed contrast wash-in., ${ }^{4,9}$ Rapid, dynamic imaging obtained after administration of contrast agents improves detection of microadenomas in comparison with postcontrast T1-weighted spin echo imaging by using the same principles. ${ }^{10,20}$ Similarly, volumetric gradient recalled echo (3DGRE) improves detection by demonstrating microadenomas as hypo-enhancing regions. ${ }^{18,28,37}$ Conversely, contrast may be retained longer in pituitary adenomas compared with normal pituitary gland.9,21 This principle underlies a theoretically complementary strategy for imaging microadenomas due to delayed contrast washout. Postcontrast FLAIR is particularly sensitive in detecting intracranial pathological entities in postcontrast images. ${ }^{12,29}$ We have previously reported that postcontrast FLAIR imaging may be useful in detecting otherwise MRI-negative pituitary microadenoma as a focus of hyperintensity. ${ }^{28}$ We hypothesized that this imaging modality increases the accuracy for MRI detection of ACTH-secreting pituitary adenomas as an adjunct sequence to the current, widely used 3DGRE MRI. In this study, we compare the performance of 3D-GRE and FLAIR sequences in 23 patients with biochemically proven $\mathrm{CD}$. We report that postcontrast FLAIR imaging may be potentially useful in detecting otherwise MRI-negative microadenomas in CD.

\section{Methods \\ Patient Population}

We retrospectively analyzed imaging, histopathological, and surgical findings in 23 patients ( 24 tumors; 16 female patients, 7 male patients) with biochemically proven CD who underwent transsphenoidal surgery (TSS) for CD at the National Institutes of Health (NIH) between 2013 and 2016 under NIH protocol 03-N-0164 (ClinicalTrials. gov; identifier NCT00060541). All patients with a working diagnosis of $\mathrm{CD}$ who underwent pituitary postcontrast FLAIR imaging were included in the current study. The protocol was approved by the Combined Neuroscience Institutional Review Board of the NIH in Bethesda, Maryland, and informed consent was obtained from all participants. The study was performed in conformance with the standards and protocols of the institutional review board.

\section{Diagnosis of CD}

Standard biochemical workup for Cushing's syndrome was done in all patients, and hypercortisolism was diag- nosed on the basis of elevations in at least 2 of the following laboratory values: 24-hour urinary free cortisol (UFC) (normal range $1.4-20 \mu \mathrm{g} / 24$ hours for 3 - to 8 -year-olds, 2.6-37 $\mu \mathrm{g} / 24$ hours for 9 - to 12 -year-olds, $4.0-56 \mu \mathrm{g} / 24$ hours for 13- to 17-year-olds, and 3.5-45.0 $\mu \mathrm{g} / 24$ hours for $\geq 18$-year-olds); late-night salivary cortisol (normal range $<100 \mathrm{ng} / \mathrm{dl}$ ); or low-dose dexamethasone suppression testing (1 mg overnight or $2 \mathrm{mg}$ over 48 hours).

Additional diagnostic workup included midnight serum cortisol (normal range $<7.5 \mu \mathrm{g} / \mathrm{dl}$ for adults, $<4 \mu \mathrm{g}$ / dl for children) and ACTH (normal range 5-46 pg/ml), and 24-hour urinary 17-hydroxycorticosteroids (17-OHS/ Cr) excretion (normal range 3-10 mg/24 hours for males and $2-6 \mathrm{mg} / 24$ hours for females). The pituitary source of $\mathrm{ACTH}$ secretion was evaluated by early-morning ACTH and cortisol levels to assess diurnal rhythmicity; an 8-mg overnight high-dose dexamethasone suppression test (HDDST); corticotropin-releasing hormone (CRH) stimulation testing; and MRI of the pituitary gland. Patients with suppression of cortisol levels on HDDST, CRHinduced elevation of ACTH and cortisol levels, and with MRI evidence of a pituitary adenoma received a diagnosis of $\mathrm{CD} .{ }^{33} \mathrm{~A}$ confirmatory IPSS procedure with CRH stimulation was performed in all patients $(n=16)$, except where HDDST and CRH stimulation testing were concordant, and MRI demonstrated a distinct adenoma $>6 \mathrm{~mm}$.

\section{Protocol for MRI}

Following confirmation of ACTH-dependent endogenous hypercortisolism, patients underwent preoperative pituitary MRI with precontrast T2-weighted, postcontrast 3D-GRE, and postcontrast FLAIR sequences. Contrast injection and 3D-GRE acquisition were simultaneously initiated, and the 3D-GRE scan acquisition time was 3 minutes. The IPSS was performed in cases in which preoperative testing was not completely congruent as a confirmatory investigation. The MRI of the pituitary gland was performed in all patients before TSS; MRI slices in coronal and sagittal orientations were obtained using a 1-mm 3D-GRE (TR $9.6 \mathrm{msec}$, TE $2.3 \mathrm{msec}, 12-\mathrm{cm}$ field of view) sequence as previously described, ${ }^{37}$ and a $3-\mathrm{mm}$ FLAIR sequence was also performed. Contiguous 1-mmthick coronal slices were acquired for 3D-GRE sequences. Coronal FLAIR scans were performed using the following parameters: TR $9000 \mathrm{msec}$, TE $95 \mathrm{msec}$, and a 12-cm field of view for which contiguous $3-\mathrm{mm}$ slices were obtained. Images were obtained on 1.5- and 3-T MRI machines (Table 1). Based on a previous study, we believe that optimal FLAIR sequence capture is 30-60 minutes after contrast injection. ${ }^{9}$

\section{Surgical Intervention}

All patients were taken to the operating room for resection of their pituitary adenoma via a microscopic sublabial submucosal transsphenoidal approach in which a dissection based on the presence of a pseudocapsule was performed by a single surgeon (P.C.). ${ }^{36}$ Diagnosis of CD was ultimately confirmed by histopathological examination of surgical specimens. Early remission from $C D$ was defined as postoperative hypocortisolemia (morning serum cortisol $<5 \mu \mathrm{g} / \mathrm{dl})$ within 5 days. ${ }^{24}$ 
TABLE 1. Demographic and clinical data in 23 patients with 24 pituitary adenomas

\begin{tabular}{|c|c|c|c|c|c|c|c|c|c|}
\hline $\begin{array}{l}\text { Case } \\
\text { No. }\end{array}$ & $\begin{array}{l}\text { Age } \\
\text { (yrs) }\end{array}$ & Sex & SPGR & FLAIR & $\begin{array}{l}\text { Tumor Found } \\
\text { at Surgical Site }\end{array}$ & $\begin{array}{c}\text { ACTH } \\
\text { Adenoma }\end{array}$ & Remission & $\begin{array}{l}\text { Time Btwn 1st Postcontrast } \\
\text { Image \& FLAIR (mins) }\end{array}$ & $\begin{array}{l}\text { MRI Field } \\
\text { Strength }(T)\end{array}$ \\
\hline 1 & 14 & $M$ & + & - & Yes & Yes & Yes & 19 & 3 \\
\hline 2 & 46 & $\mathrm{~F}$ & ++ & ++ & Yes & Yes & Yes & 23 & 3 \\
\hline 3 & 20 & $F$ & + & - & Yes & Yes & Yes & 19 & 3 \\
\hline 4 & 18 & $\mathrm{~F}$ & ++ & ++ & Yes & Yes & Yes & 25 & 3 \\
\hline 5 & 34 & $M$ & ++ & ++ & Yes & Yes & Yes & 23 & 1.5 \\
\hline 6 & 19 & $\mathrm{~F}$ & ++ & - & Yes & Yes & Yes & 28 & 3 \\
\hline 7 & 24 & M & - & ++ & No & No & No & 21 & 3 \\
\hline 8 & 42 & $\mathrm{~F}$ & + & - & Yes & Yes & Yes & 23 & 1.5 \\
\hline $9 a$ & 45 & $\mathrm{~F}$ & ++ & - & Yes & No & No & 25 & 1.5 \\
\hline $9 b$ & 45 & $\mathrm{~F}$ & - & ++ & Yes & Yes & Yes & 25 & 1.5 \\
\hline 10 & 58 & $\mathrm{~F}$ & + & - & No & Yes & Yes & 32 & 3 \\
\hline 11 & 9 & $\mathrm{~F}$ & - & ++ & Yes & Yes & Yes & 30 & 3 \\
\hline 12 & 19 & M & - & ++ & Yes & Yes & Yes & 30 & 3 \\
\hline 13 & 17 & $M$ & ++ & - & Yes & Yes & Yes & 31 & 3 \\
\hline 14 & 34 & $\mathrm{~F}$ & +++ & +++ & Yes & Yes & Yes & 18 & 1.5 \\
\hline 15 & 10 & $\mathrm{~F}$ & + & - & Yes & Yes & Yes & 11 & 3 \\
\hline 16 & 16 & $F$ & ++ & - & Yes & Yes & Yes & 13 & 1.5 \\
\hline 17 & 55 & $\mathrm{~F}$ & ++ & ++ & Yes & Yes & Yes & 12 & 3 \\
\hline 18 & 52 & $\mathrm{M}$ & +++ & +++ & Yes & Yes & No & 15 & 3 \\
\hline 19 & 18 & $\mathrm{~F}$ & ++ & - & Yes & Yes & Yes & 12 & 3 \\
\hline 20 & 9 & $M$ & - & ++ & Yes & Yes & Yes & 15 & 3 \\
\hline 21 & 46 & $\mathrm{~F}$ & +++ & +++ & Yes & Yes & Yes & 15 & 1.5 \\
\hline 22 & 27 & $\mathrm{~F}$ & + & - & Yes & Yes & No & 26 & 1.5 \\
\hline 23 & 43 & $\mathrm{~F}$ & ++ & ++ & Yes & Yes & Yes & 33 & 1.5 \\
\hline
\end{tabular}

Radiological results were independently reported as negative (-), possible microadenoma (+), definitive microadenoma (++), and macroadenoma (+++) for 3D-GRE (SPGR) and postcontrast FLAIR sequences.

\section{Data Capture and Analysis}

Two experienced radiologists (N.P. and J.S.) reviewed the coronal postcontrast 3D-GRE and postcontrast FLAIR images blindly and independently of each other by using a randomized patient list on 2 different days. The first radiologist (N.P.) assessed 3D-GRE followed by FLAIR sequences. To improve data capture and analysis, the order of MRI sequences was randomized for our second neuroradiologist (J.S.). Both radiologists recorded the presence, size, and location of lesions-hypointensities on postcontrast 3D-GRE, and hyperintensities on postcontrast FLAIR sequences. In instances in which findings were equivocal, adenomas were characterized as "possible" (Table 1). These findings were then compared with the surgical findings of the neurosurgeon, which were recorded graphically in the handwritten postoperative record of the pituitary gland denoting the size and location of the adenoma. Histopathology results were then examined, and location concordance of ACTH-positive pituitary adenomas to the imaging and/or surgical findings was noted. A true-positive MRI finding of adenoma was assigned only if the histopathological finding of an ACTH-positive adenoma was location concordant with the imaging findings. Absence of this concordance led to false-positive or false-negative designations. A blinded analysis of histo- pathological findings to assess for differences in tissue cohesiveness and vascular density in pituitary adenomas was performed by an experienced neuropathologist (A.R.).

\section{Statistical Analysis}

Sensitivity, specificity, positive predictive value (PPV), and negative predictive value were calculated by comparing the imaging sequence data to the location-concordant histopathological finding as the gold standard. For purposes of statistical analysis, cases with equivocal radiological findings were classified as negative.

Statistical significance was determined by unpaired ttest using the GraphPad Prism version 6.0 software program when comparing workup endocrinology laboratory values and clinical data for FLAIR-positive and -negative patient groups. Fisher's exact test was performed when comparing tissue cohesiveness and vascular density for FLAIR-positive and -negative patient groups. A p value of 0.05 was used to assess statistical significance. An outlier analysis was performed using the robust regression and outlier removal (or ROUT) method, with a maximum false discovery rate (or Q) set at $1 \%$. Outliers identified using this method were discarded.

The degree of observed agreement between the 2 radiologists was determined by cross-tabulating radiological results. 
A kappa value was calculated using previous guidelines. ${ }^{14}$ For interpretation, we used the following ranges: kappa values from 0.0 to 0.2 indicated slight agreement, 0.21 to 0.40 indicated fair agreement, 0.41 to 0.60 indicated moderate agreement, 0.61 to 0.80 indicated substantial agreement, and 0.81 to 1.0 indicated almost-perfect or perfect agreement. ${ }^{14}$

\section{Results \\ Demographic Data}

Twenty-three consecutive patients (9-58 years old, 16 females and 7 males) were included in this study. Adrenocorticotropic hormone-dependent hypercortisolemia was demonstrated in all individuals with the following findings: 1) elevated 24-hour UFC (mean 694.5 $\pm 1335.2 \mu \mathrm{g} / 24$ hours, normal range 1.4-20 $\mu \mathrm{g} / 24$ hours for 3- to 8-yearolds, 2.6-37 $\mu \mathrm{g} / 24$ hours for 9- to 12-year-olds, 4.0-56 $\mu \mathrm{g} / 24$ hours for 13 - to 17 -year-olds, and 3.5-45.0 $\mu \mathrm{g} / 24$ hours for $\geq 18$-year-olds); 2 ) elevated midnight serum cortisol (mean $22 \pm 13.4 \mu \mathrm{g} / \mathrm{dl}$, normal range $<7.5 \mu \mathrm{g} / \mathrm{dl}$ ); and 3) lack of suppression of midnight plasma ACTH (mean $48.2 \pm 29.5 \mathrm{pg} / \mathrm{ml}$, normal range 5-46 pg/ml) (Table 1).

\section{Radiological Results}

On postcontrast 3D-GRE, adenomas were detected as lesions that were hypointense compared with the surrounding normal pituitary parenchyma. ${ }^{37}$ On postcontrast FLAIR, the lesions were hyperintense, presumably due to delayed contrast washout. ${ }^{28}$ We first compared the standalone accuracy of 3D-GRE and FLAIR sequences in detecting pituitary microadenomas.

In the 23 patients (24 tumors) screened with 3D-GRE MRI sequence, 3 macroadenomas ( $>10 \mathrm{~mm}), 10$ distinct microadenomas, and 6 possible microadenomas were reported (Table 1). In 1 patient, 2 pituitary adenomas were found (Cases 9a and 9b); of those, 3D-GRE detected 1 adenoma. Using 3D-GRE images, the radiologist's read was true positive in 18/24 (75\%) (location-concordant, histopathologically confirmed pituitary adenomas); false negative in 4/24 (17\%; MRI-negative CD); and false positive in $1 / 24$ (4\%) cases. Using postcontrast FLAIR sequence only, the radiologist's read was true positive in 12/24 (50\%), false negative in 10/24 (42\%), and false positive in 1/24 (4\%) cases. These results confirm the superiority of 3D-GRE as a stand-alone imaging modality. Overall, of the 24 pituitary adenomas, 18 (75\%; sensitivity 82\%, PPV 95\%) were found on 3D-GRE, and 12 (50\%; sensitivity 55\%, PPV 92\%) were correctly identified on FLAIR. The stand-alone specificity of 3D-GRE and FLAIR was similar (50\%).

Because cysts may appear hyperintense on postcontrast FLAIR due to increased fluid content or contrast accumulation, ${ }^{19,46} \mathrm{~T} 2$-weighted sequences were used to screen for cysts within the pituitary gland or the adenoma (Fig. 1). We then tested the utility of FLAIR as a complementary tool to 3D-GRE. Interestingly, all 5 patients with negative 3D-GRE MRI displayed a distinct focus of FLAIR enhancement (Fig. 2). Four of those 5 cases (80\%) had location-concurrent positive histopathological findings and achieved postsurgical biochemical remission. The remaining patient did not achieve remission from $\mathrm{CD}$ despite subtotal hypophysectomy. No intraoperative tumor was found; therefore a subtotal hypophysectomy was performed that did not include the FLAIR hyperintensity on the left superior aspect of the pituitary gland (Fig. 3). This patient's histopathology report was negative for ACTHpositive pituitary adenoma (Table 1; Case 7).

The interobserver agreement for adenoma detection using 3D-GRE sequences was moderate $(\kappa=0.59)$ between our 2 independent neuroradiologists. Compared with N.P., J.S. identified 2 additional 3D-GRE lesions, 1 of which was location concordant. We believe that an increase in positive reads between the neuroradiologists could reflect confirmation bias due to the established diagnosis of CD. Interestingly, there was a significant overlap for patients whose 3D-GRE screenings were negative but who had a positive FLAIR. Both radiologists identified Cases 7, 9b, and 11 as 3D-GRE negative and FLAIR positive. Our second radiologist (J.S.) identified an additional 3D-GREnegative and FLAIR-positive lesion that was not location concordant. When assessing FLAIR by itself, the interrater variability showed a fair agreement $(\kappa=0.32)$, illustrating the need to develop further strategies to standardize acquisition and interpretation of postcontrast delayed FLAIR in pituitary imaging.

\section{Surgical Treatment and Outcome}

All 23 patients underwent TSS. For 2 patients, the surgeon failed to identify a pituitary adenoma in the surgical field (Table 1; Cases 7 and 10). Both of these patients had preoperative testing including IPSS results consistent with CD (Supplemental Table 1). In Case 7, because the suspected adenoma was not localized on the 3D-GRE screening MRI, a subtotal hypophysectomy ${ }^{26,34,42}$ was therefore performed, during which $75 \%$ of the anterior pituitary was removed. Histopathological investigation failed to reveal an ACTH-containing pituitary adenoma, and the patient did not achieve biochemical remission. Because subtotal hypophysectomy is typically the final surgical intervention directed at the sella in $\mathrm{CD}$, the patient did not receive an additional TSS. In retrospect, the resection did not include the region of FLAIR hyperintensity (Fig. 3). In Case 10, although no tumor was found during surgery, the patient underwent a subtotal hypophysectomy, which led to postsurgical remission. Histopathological investigation confirmed the presence of an ACTH-secreting adenoma within the submitted hypophysectomy specimen. Two other patients did not achieve remission from $C D$ due to residual tumor-one with a macroadenoma with invasion of cavernous sinus and sphenoid bone (Case 18), and the second patient (Case 22) with a microadenoma and significant invasion of the dura and extension of the tumor into the right cavernous sinus.

For all other cases (19 patients, 20 adenomas), a location-concordant (with either 3D-GRE or FLAIR findings), histopathologically proven pituitary adenoma was found. As previously reported, in 1 patient, 2 pituitary adenomas were found with different imaging characteristics upon reviewing 3D-GRE versus FLAIR sequences (Table 1; Cases $9 \mathrm{a}$ and $9 \mathrm{~b}$ ). ${ }^{28}$ This patient initially underwent TSS on the left side of the pituitary gland due to a positive 3DGRE MRI finding. Due to lack of biochemical remission and histopathological findings revealing null-cell adeno- 


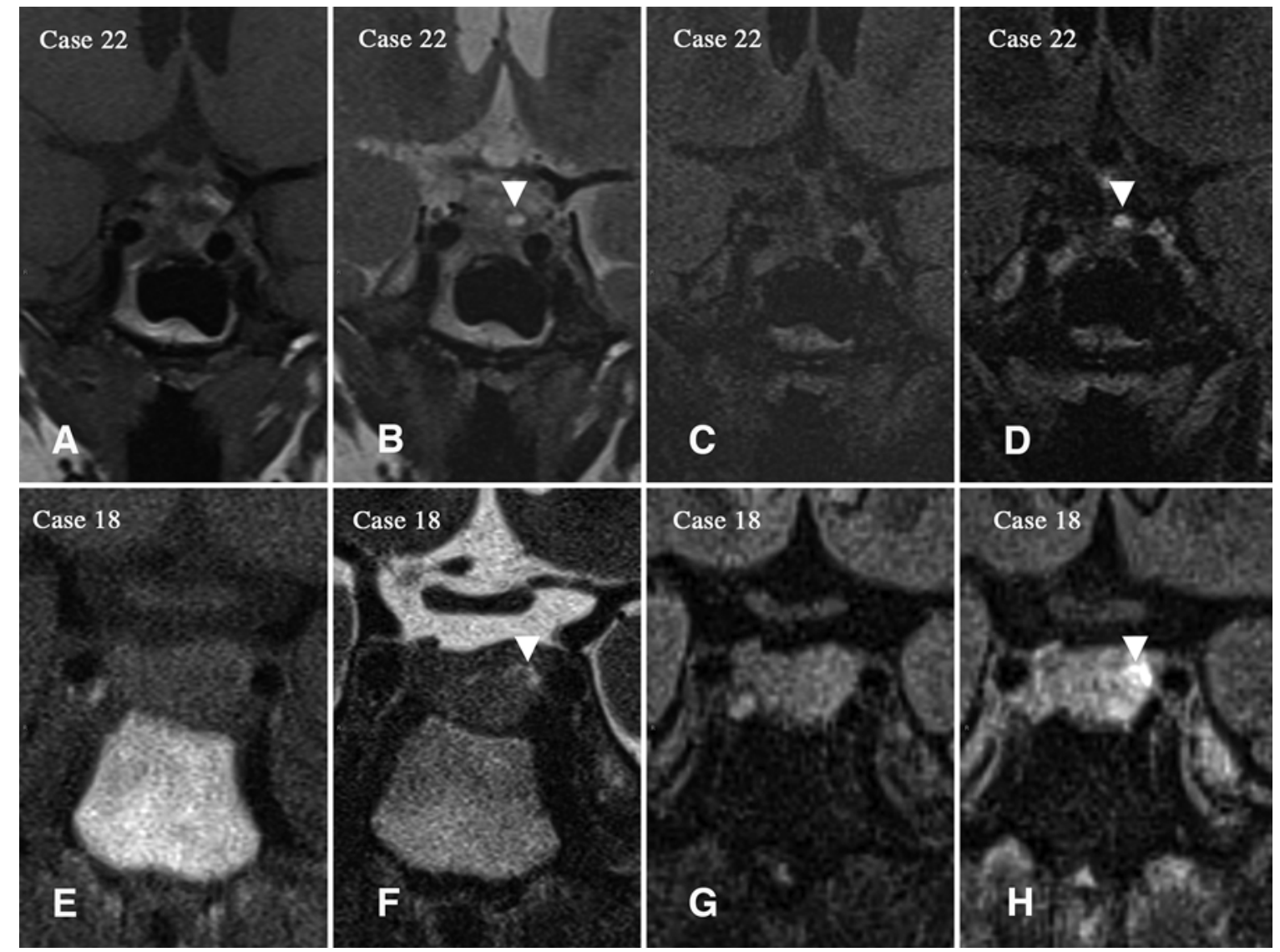

FIG. 1. Noncontrast T1-weighted ( $A$ and $E$ ) and T2-weighted images ( $B$ and $F)$ were useful in detecting pituitary (B, white arrowhead) and intratumoral ( $F$, white arrowhead) cysts. Corresponding regions were hyperintense on postcontrast FLAIR images (white arrowheads in $\mathbf{D}$ and $\mathbf{H}$ ), whereas they were isointense on precontrast FLAIR images ( $\mathbf{C}$ and $\mathbf{G})$.

ma, the patient returned to the operating room for an early reoperation on postoperative Day 7. A second pituitary adenoma was found on the right side, corresponding to hyperintensity on postcontrast FLAIR imaging. This adenoma was subsequently confirmed by histopathological investigation as an ACTH-secreting adenoma, and laboratory data confirmed remission of CD.

\section{Determinants of FLAIR Hyperintensity}

We then compared the preoperative laboratory test val-
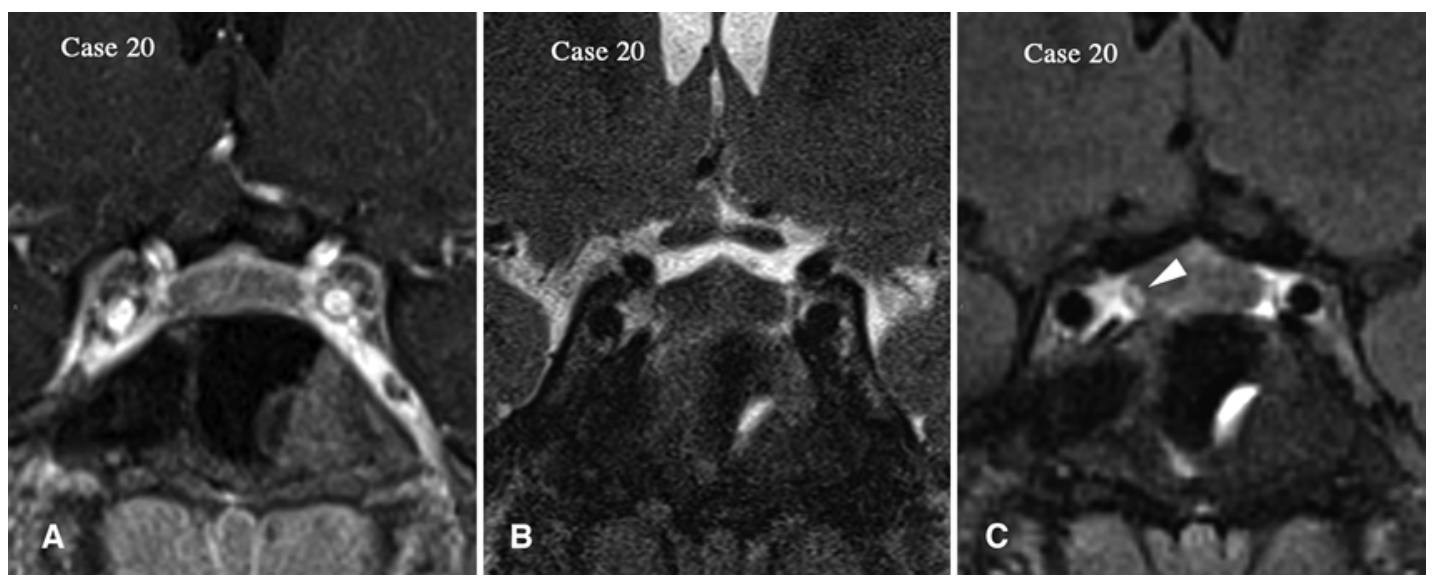

FIG. 2. Volumetric spoiled gradient-recalled acquisition in the steady state, a 3D gradient echo technique, fails to demonstrate a pituitary microadenoma (A). The T2-weighted sequence is also negative and rules out any cystic or necrotic areas of the pituitary gland (B). Postcontrast FLAIR image reveals marked contrast enhancement in the ACTH-positive adenoma in the far-right lateral aspect of the gland (C, white arrowhead). 

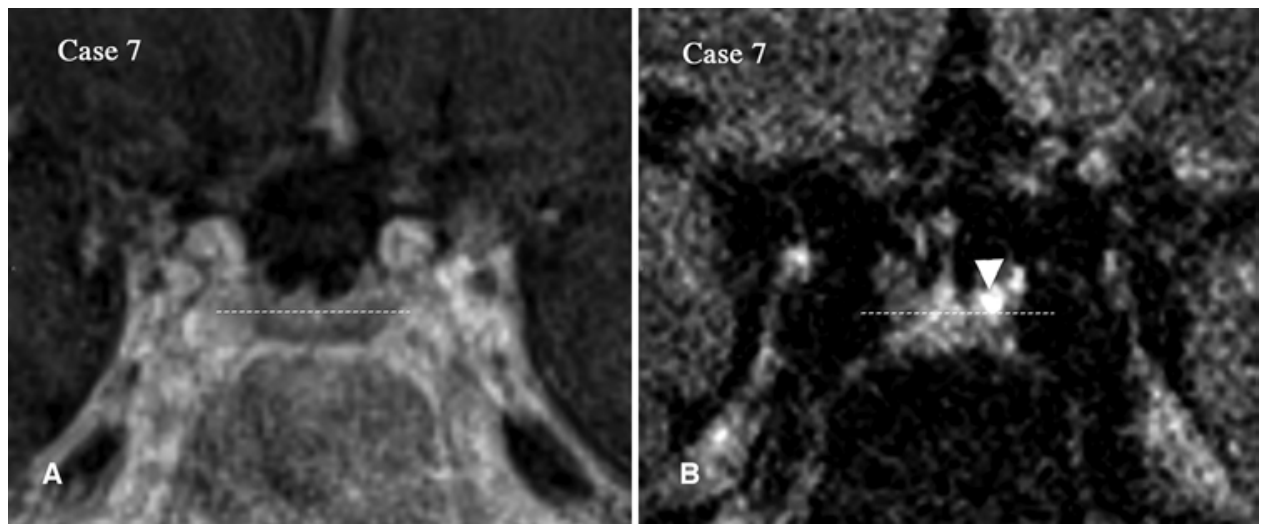

FIG. 3. Postcontrast 3D-GRE image fails to demonstrate a pituitary adenoma (A). Delayed postcontrast FLAIR image reveals marked contrast enhancement in the left aspect of the gland (B, white arrowhead). Resection did not include the hyperintense area on the FLAIR image, as shown by the delineation of the subtotal hypophysectomy (dotted line). The surgical specimen did not contain pituitary adenoma, and the patient did not achieve remission from CD.

ues for patients with FLAIR-positive $(\mathrm{n}=13)$ and FLAIRnegative $(\mathrm{n}=11)$ adenomas. We found a statistically significant elevation of midnight ACTH in the FLAIR-positive group $(63.08 \mathrm{pg} / \mathrm{ml}$ vs $34.72 \mathrm{pg} / \mathrm{ml}$, difference between means $-28.35 \pm 11.49 \mathrm{pg} / \mathrm{ml}, 95 \%$ CI -52.40 to -4.308 $\mathrm{pg} / \mathrm{ml} ; \mathrm{p}=0.02$ ). Despite increased plasma ACTH levels, we found no difference in the UFC $(957.6 \mu \mathrm{g} / 24$ hours vs $383.5 \mu \mathrm{g} / 24$ hours, $\mathrm{p}=0.30$ ) and midnight cortisol $(26.58 \mu \mathrm{g} / \mathrm{dl}$ vs $16.97 \mu \mathrm{g} / \mathrm{dl}, \mathrm{p}=0.08)$ (Fig. 4). Similarly, no statistically significant difference was found between the 2 groups in their responses to CRH-stimulation testing (cortisol: $134.4 \%$ vs $82.97 \%, p=0.22$; ACTH: $323.4 \%$ vs $215.4 \%, \mathrm{p}=0.39)$ or response to HDDST $(-43.31 \%$ vs $-73.75 \%, \mathrm{p}=0.07$ ). We then tested whether delay (mean $21.24 \pm 6.9$ minutes, range 11-33 minutes) in acquisition of FLAIR imaging following intravenous contrast injection affected the appearance of lesions as hyperintensities on FLAIR sequences. We found no difference (21.92 minutes vs 21.73 minutes, $\mathrm{p}=0.95$ ) between the 2 groups (Supplemental Table 1). The patients had no difference in age (33.4 years vs 26 years, $\mathrm{p}=0.26)$, sex $(61.5 \%$ female vs $81.8 \%$ female, $\mathrm{p}=0.29)$, time from onset of $\mathrm{CD}(55.6$ months vs 23.3 months, $p=0.12$ ), or tumor volumes (780.1 $\mathrm{mm}^{3}$ vs $87.4 \mathrm{~mm}^{3}, \mathrm{p}=0.26$ ). Results from blinded analysis of histopathological findings did not reveal significantly increased vasculature $(p=1)$ or decreased tissue cohesiveness $(p=1)$ in the FLAIR-positive when compared with the FLAIR-negative group.

\section{Discussion}

Accurate diagnosis of $\mathrm{CD}$ requires a stepwise approach of laboratory evaluations and imaging studies. ${ }^{33}$ In patients found to have ACTH-dependent Cushing's syndrome, an MRI study of the pituitary with contrast administration is performed. The MRI findings may be negative for up to $20 \%-50 \%$ of patients with $\mathrm{CD},{ }^{26}$ with localization of microadenoma leading to less extensive exploration ${ }^{34}$ and arguably improved outcomes. ${ }^{2,41}$ Microadenomas are typically visualized with a gadolinium-based agent as hypointense lesions on early postcontrast imaging. ${ }^{9}$ The $3 \mathrm{D}^{-G R E^{37,48}}$ and dynamic imaging protocols may improve sensitivity compared with conventional spin echo imaging by optimizing early postcontrast imaging to maximize this difference in contrast uptake. ${ }^{11,45}$ Conversely, pituitary tumors may retain contrast longer than normal pituitary parenchyma. ${ }^{9,10,20}$ Because MR imaging-invisible adenomas in CD are small $(<5 \mathrm{~mm}){ }_{17}^{17}$ sensitive imaging modalities are needed to detect retained contrast. Postcontrast FLAIR imaging is a sensitive modality for extraaxial contrast enhancement in numerous intracranial pathological entities, including leptomeningeal metastases, meningitis, and subdural hematomas. ${ }^{12,29}$ We have previously reported the utility of postcontrast FLAIR in detecting pituitary adenoma (Table 1; Cases 9a and 9b), presumably due to delayed contrast washout. ${ }^{28}$

In this report, although FLAIR imaging had a significantly lower sensitivity compared with 3D-GRE (82\% vs $55 \%$ ), the true value of FLAIR may be as a complementary tool to 3D-GRE. All 5 patients with negative findings on 3D-GRE MRI in this series displayed a distinct focus of FLAIR enhancement (Fig. 2). In 4 of them, an adenoma was found during surgical exploration and was confirmed by histopathological examination. The region of FLAIR hyperintensity was not included in the resected specimen in the 1 patient with persistent hypercortisolism after hemihypophysectomy for which histopathological findings were negative, as expected (Table 1; Case 7). In addition, when comparing radiological results between our 2 neuroradiologists, there was a significant overlap for cases that were 3D-GRE negative but FLAIR positive (Cases 7, $9 \mathrm{~b}$, and 11).

With evidence from this case series, we propose to add FLAIR sequences to the current diagnostic imaging protocol for CD (Fig. 5). Acquisition of 3D-GRE and T2-weighted images will allow identification and screening of cystic and necrotic regions. Addition of FLAIR sequences could supplement detection of occult adenomas in $\mathrm{CD}$. If validated in larger studies, FLAIR imaging could complement 3D-GRE or dynamic imaging prior to invasive testing such as IPSS. ${ }^{30,43,44}$ Such a strategy is feasible, considering the very low incidence $(<3.3 \%)$ of so-called incidentalomas in $\mathrm{CD},{ }^{16,28,39,40}$ compared with up to $30 \%$ found in the general 


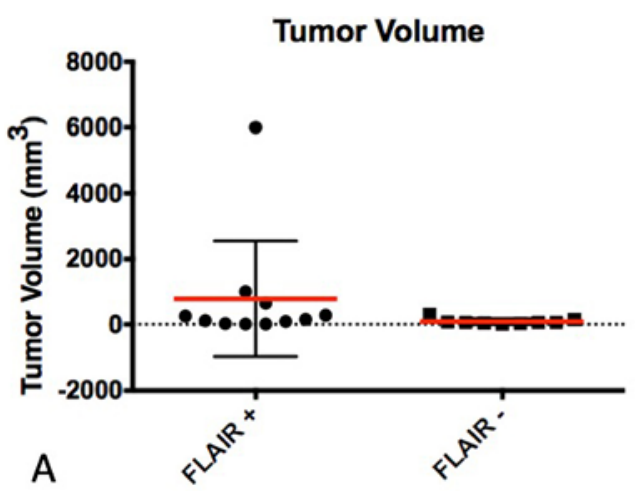

Midnight Cortisol

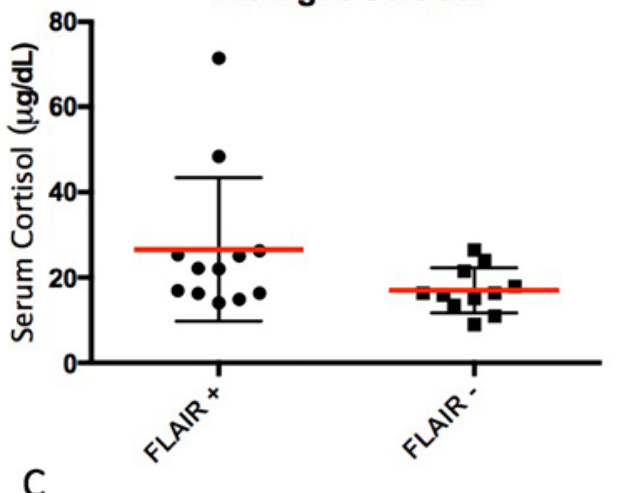

Time to FLAIR images

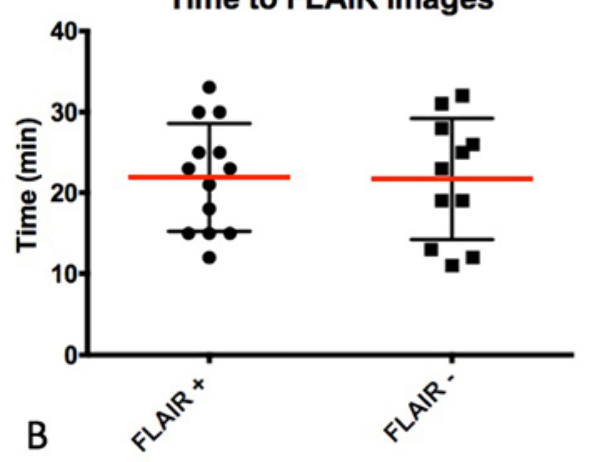

Midnight ACTH

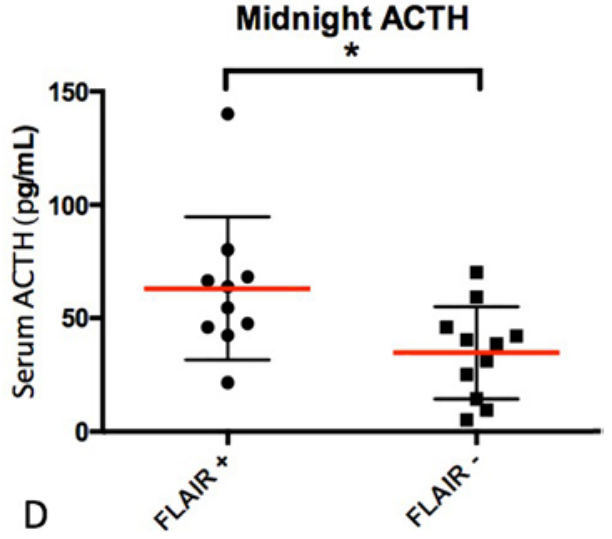

FIG. 4. Graphs showing comparisons of FLAIR-positive versus FLAIR-negative images for tumor volume (A), time to acquisition of FLAIR images following intravenous contrast injection (B), midnight cortisol (C), and midnight ACTH (D). Horizontal bars represent the mean $\pm S D$. ${ }^{*} p \leq 0.05$. Figure is available in color online only.

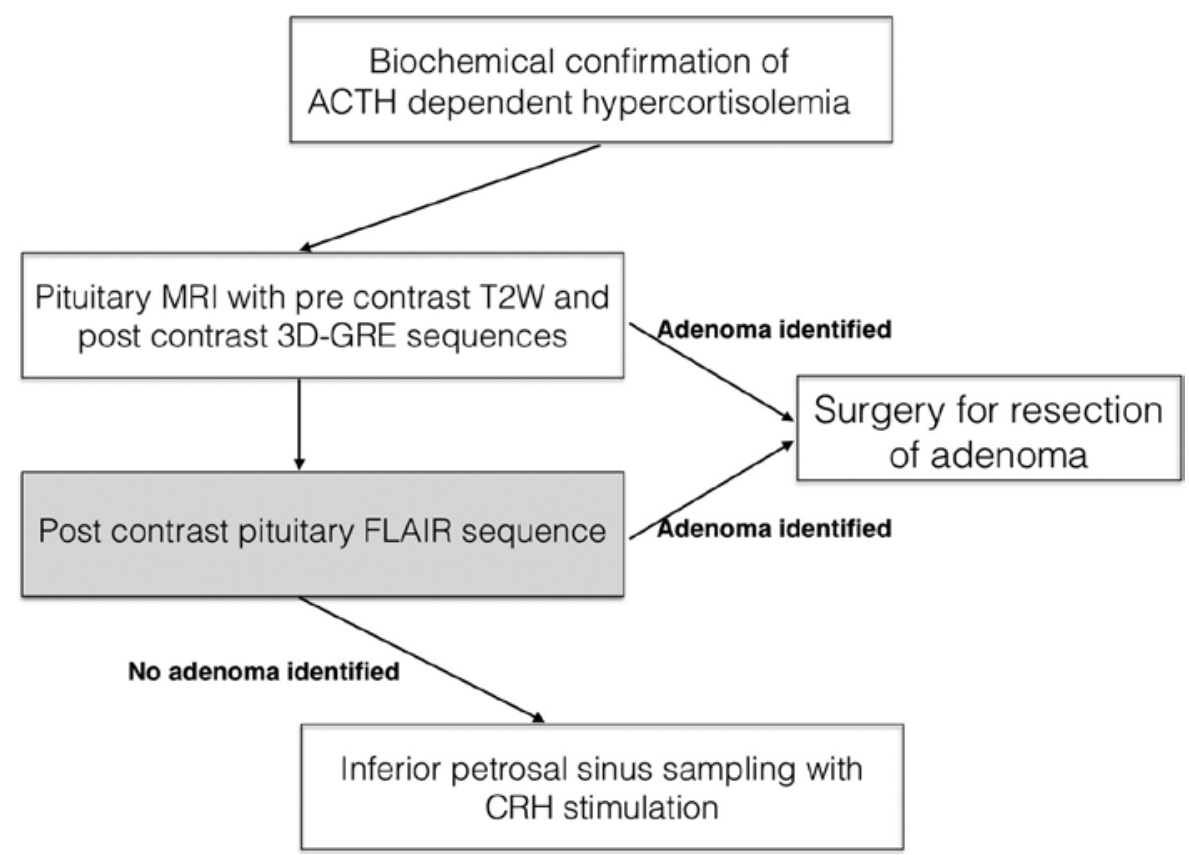

FIG. 5. Flow chart showing potential pituitary imaging protocol for CD. Patients with biochemical workup suggestive of $C D$ first undergo pituitary MRI with precontrast T2-weighted (T2W) and postcontrast 3D-GRE sequences. We propose adding a postcontrast FLAIR sequence (gray box) for patients who were screened with T2-weighted and postcontrast 3D-GRE sequences, and in whom no adenomas were identified. The T2-weighted sequences assist in detecting cysts that may also appear hyperintense on postcontrast FLAIR imaging. 
population. ${ }^{1,13,31}$ Although there was an apparent disagreement in FLAIR sequence results between the 2 radiologists, this highlights the need for further standardization of reading techniques as well as protocol. We are now designing a new clinical protocol to address this and hope to be performing a prospective blinded study.

The biological phenomenon underlying the delayed postcontrast retention in some adenomas is uncertain. We did find elevated plasma ACTH levels in patients with FLAIR-positive lesions (Fig. 4). The significance of these findings remains unknown. No other historical (time of onset of CD), structural (tumor volume), or biochemical differences were found between groups. Histopathological correlates of FLAIR-positive lesions are currently unknown. Although pituitary adenomas tend to have a lower total microvascular volume when compared with normal pituitary, ${ }^{47}$ the tumor vessels tend to be larger. ${ }^{38}$ The difference in the size of vasculature ${ }^{38}$ may underlie increased contrast retention 22,27 in adenomas detected on FLAIR images. Alternatively, the FLAIR-positive signal may come from distortion of the tissue architecture of microadenomas. The FLAIR-positive microadenomas may possess looser interstitium, causing a delayed washout of contrast that is seen as increased enhancement on imaging. Results from our blinded analysis of histopathological findings, however, did not reveal significantly increased vasculature or decreased tissue cohesiveness in FLAIR-positive when compared with the FLAIR-negative group, suggesting other potential underlying biological phenomena.

\section{Conclusions}

This study suggests that delayed contrast washout in microadenomas may be detected as FLAIR hyperintensity in otherwise MRI-negative CD cases. We propose adding postcontrast FLAIR sequences to complement high-resolution 3D-GRE for surgical planning in patients with CD.

\section{Acknowledgments}

This study was supported by the Intramural Research Programs of the NINDS, the Eunice Kennedy Shriver National Institute of Child Health and Human Development, and the NIH Clinical Center in Bethesda, MD.

\section{References}

1. Bancos I, Natt N, Murad MH, Montori VM: Evidence-based endocrinology: illustrating its principles in the management of patients with pituitary incidentalomas. Best Pract Res Clin Endocrinol Metab 26:9-19, 2012

2. Biller BMK, Grossman AB, Stewart PM, Melmed S, Bertagna X, Bertherat J, et al: Treatment of adrenocorticotropindependent Cushing's syndrome: a consensus statement. J Clin Endocrinol Metab 93:2454-2462, 2008

3. Bochicchio D, Losa M, Buchfelder M: Factors influencing the immediate and late outcome of Cushing's disease treated by transsphenoidal surgery: a retrospective study by the European Cushing's Disease Survey Group. J Clin Endocrinol Metab 80:3114-3120, 1995

4. Bonneville JF, Cattin F, Moussa-Bacha K, Portha C: Dynamic computed tomography of the pituitary gland: the "tuft sign". Radiology 149:145-148, 1983

5. Chittiboina P, Montgomery BK, Millo C, Herscovitch P, Lonser RR: High-resolution ${ }^{18} \mathrm{~F}$-fluorodeoxyglucose positron emission tomography and magnetic resonance imaging for pituitary adenoma detection in Cushing disease. J Neurosurg 122:791-797, 2015

6. Chowdhury IN, Sinaii N, Oldfield EH, Patronas N, Nieman LK: A change in pituitary magnetic resonance imaging protocol detects ACTH-secreting tumours in patients with previously negative results. Clin Endocrinol (Oxf) 72:502-506, 2010

7. Davis PC, Hoffman JC Jr, Malko JA, Tindall GT, Takei Y, Avruch L, et al: Gadolinium-DTPA and MR imaging of pituitary adenoma: a preliminary report. AJNR Am J Neuroradiol 8:817-823, 1987

8. de Herder WW, Uitterlinden P, Pieterman H, Tanghe HL, Kwekkeboom DJ, Pols HA, et al: Pituitary tumour localization in patients with Cushing's disease by magnetic resonance imaging. Is there a place for petrosal sinus sampling? Clin Endocrinol (Oxf) 40:87-92, 1994

9. Dwyer AJ, Frank JA, Doppman JL, Oldfield EH, Hickey AM, Cutler GB, et al: Pituitary adenomas in patients with Cushing disease: initial experience with Gd-DTPA-enhanced MR imaging. Radiology 163:421-426, 1987

10. Finelli DA, Kaufman B: Varied microcirculation of pituitary adenomas at rapid, dynamic, contrast-enhanced MR imaging. Radiology 189:205-210, 1993

11. Friedman TC, Zuckerbraun E, Lee ML, Kabil MS, Shahinian $\mathrm{H}$ : Dynamic pituitary MRI has high sensitivity and specificity for the diagnosis of mild Cushing's syndrome and should be part of the initial workup. Horm Metab Res 39:451-456, 2007

12. Goo HW, Choi CG: Post-contrast FLAIR MR imaging of the brain in children: normal and abnormal intracranial enhancement. Pediatr Radiol 33:843-849, 2003

13. Hall WA, Luciano MG, Doppman JL, Patronas NJ, Oldfield EH: Pituitary magnetic resonance imaging in normal human volunteers: occult adenomas in the general population. Ann Intern Med 120:817-820, 1994

14. Hallgren KA: Computing inter-rater reliability for observational data: an overview and tutorial. Tutor Quant Methods Psychol 8:23-34, 2012

15. Hofmann BM, Hlavac M, Martinez R, Buchfelder M, Müller OA, Fahlbusch R: Long-term results after microsurgery for Cushing disease: experience with 426 primary operations over 35 years. J Neurosurg 108:9-18, 2008

16. Iacovazzo D, Bianchi A, Lugli F, Milardi D, Giampietro A, Lucci-Cordisco E, et al: Double pituitary adenomas. Endocrine 43:452-457, 2013

17. Jagannathan J, Smith R, DeVroom HL, Vortmeyer AO, Stratakis CA, Nieman LK, et al: Outcome of using the histological pseudocapsule as a surgical capsule in Cushing disease. $\mathbf{J}$ Neurosurg 111:531-539, 2009

18. Kasaliwal R, Sankhe SS, Lila AR, Budyal SR, Jagtap VS, Sarathi V, et al: Volume interpolated 3D-spoiled gradient echo sequence is better than dynamic contrast spin echo sequence for MRI detection of corticotropin secreting pituitary microadenomas. Clin Endocrinol (Oxf) 78:825-830, 2013

19. Kubota T, Yamada K, Kizu O, Hirota T, Ito H, Ishihara K, et al: Relationship between contrast enhancement on fluidattenuated inversion recovery MR sequences and signal intensity on T2-weighted MR images: visual evaluation of brain tumors. J Magn Reson Imaging 21:694-700, 2005

20. Kucharczyk W, Bishop JE, Plewes DB, Keller MA, George S: Detection of pituitary microadenomas: comparison of dynamic keyhole fast spin-echo, unenhanced, and conventional contrast-enhanced MR imaging. AJR Am J Roentgenol 163:671-679, 1994

21. Kucharczyk W, Davis DO, Kelly WM, Sze G, Norman D, Newton TH: Pituitary adenomas: high-resolution MR imaging at $1.5 \mathrm{~T}$. Radiology 161:761-765, 1986

22. Lee EK, Lee EJ, Kim S, Lee YS: Importance of contrast- 
enhanced fluid-attenuated inversion recovery magnetic resonance imaging in various intracranial pathologic conditions. Korean J Radiol 17:127-141, 2016

23. Lee HB, Kim ST, Kim HJ, Kim KH, Jeon P, Byun HS, et al: Usefulness of the dynamic gadolinium-enhanced magnetic resonance imaging with simultaneous acquisition of coronal and sagittal planes for detection of pituitary microadenomas. Eur Radiol 22:514-518, 2012

24. Lindsay JR, Oldfield EH, Stratakis CA, Nieman LK: The postoperative basal cortisol and CRH tests for prediction of long-term remission from Cushing's disease after transsphenoidal surgery. J Clin Endocrinol Metab 96:2057-2064, 2011

25. Liu C, Lo JC, Dowd CF, Wilson CB, Kunwar S, Aron DC, et al: Cavernous and inferior petrosal sinus sampling in the evaluation of ACTH-dependent Cushing's syndrome. Clin Endocrinol (Oxf) 61:478-486, 2004

26. Lonser RR, Wind JJ, Nieman LK, Weil RJ, DeVroom HL, Oldfield EH: Outcome of surgical treatment of 200 children with Cushing's disease. J Clin Endocrinol Metab 98:892901, 2013

27. Mathews VP, Caldemeyer KS, Lowe MJ, Greenspan SL, Weber DM, Ulmer JL: Brain: gadolinium-enhanced fast fluid-attenuated inversion-recovery MR imaging. Radiology 211:257-263, 1999

28. Mehta GU, Montgomery BK, Raghavan P, Sharma S, Nieman LK, Patronas N, et al: Different imaging characteristics of concurrent pituitary adenomas in a patient with Cushing's disease. J Clin Neurosci 22:891-894, 2015

29. Melhem ER, Bert RJ, Walker RE: Usefulness of optimized gadolinium-enhanced fast fluid-attenuated inversion recovery MR imaging in revealing lesions of the brain. AJR Am J Roentgenol 171:803-807, 1998

30. Miller DL, Doppman JL, Peterman SB, Nieman LK, Oldfield EH, Chang R: Neurologic complications of petrosal sinus sampling. Radiology 185:143-147, 1992

31. Molitch ME: Pituitary tumours: pituitary incidentalomas. Best Pract Res Clin Endocrinol Metab 23:667-675, 2009

32. Moshang T Jr: Cushing's disease, 70 years later... and the beat goes on. J Clin Endocrinol Metab 88:31-33, 2003

33. Nieman LK, Biller BMK, Findling JW, Newell-Price J, Savage MO, Stewart PM, et al: The diagnosis of Cushing's syndrome: an Endocrine Society Clinical Practice Guideline. J Clin Endocrinol Metab 93:1526-1540, 2008

34. Oldfield EH: Surgical management of Cushing's disease: a personal perspective. Clin Neurosurg 58:13-26, 2011

35. Oldfield EH, Doppman JL, Nieman LK, Chrousos GP, Miller DL, Katz DA, et al: Petrosal sinus sampling with and without corticotropin-releasing hormone for the differential diagnosis of Cushing's syndrome. N Engl J Med 325:897-905, 1991

36. Oldfield EH, Vortmeyer AO: Development of a histological pseudocapsule and its use as a surgical capsule in the excision of pituitary tumors. J Neurosurg 104:7-19, 2006

37. Patronas N, Bulakbasi N, Stratakis CA, Lafferty A, Oldfield EH, Doppman J, et al: Spoiled gradient recalled acquisition in the steady state technique is superior to conventional postcontrast spin echo technique for magnetic resonance imaging detection of adrenocorticotropin-secreting pituitary tumors. J Clin Endocrinol Metab 88:1565-1569, 2003

38. Perez-Millan MI, Berner SI, Luque GM, De Bonis C, Sevlever G, Becu-Villalobos D, et al: Enhanced nestin expression and small blood vessels in human pituitary adenomas. Pituitary 16:303-310, 2013

39. Ratliff JK, Oldfield EH: Multiple pituitary adenomas in Cushing's disease. J Neurosurg 93:753-761, 2000

40. Rotondo F, Khatun N, Scheithauer BW, Horvath E, Marotta TR, Cusimano M, et al: Unusual double pituitary adenoma: a case report. Pathol Int 61:42-46, 2011

41. Salenave S, Gatta B, Pecheur S, San-Galli F, Visot A, Lasjaunias $\mathrm{P}$, et al: Pituitary magnetic resonance imaging findings do not influence surgical outcome in adrenocorticotropin-secreting microadenomas. J Clin Endocrinol Metab 89:33713376, 2004

42. Semple PL, Vance ML, Findling J, Laws ERJ Jr: Transsphenoidal surgery for Cushing's disease: outcome in patients with a normal magnetic resonance imaging scan. Neurosurgery 46:553-559, 2000

43. Seyer H, Honegger J, Schott W, Küchle M, Huk WJ, Fahlbusch R, et al: Raymond's syndrome following petrosal sinus sampling. Acta Neurochir (Wien) 131:157-159, 1994

44. Sturrock ND, Jeffcoate WJ: A neurological complication of inferior petrosal sinus sampling during investigation for Cushing's disease: a case report. J Neurol Neurosurg Psychiatry 62:527-528, 1997

45. Tabarin A, Laurent F, Catargi B, Olivier-Puel F, Lescene R, Berge J, et al: Comparative evaluation of conventional and dynamic magnetic resonance imaging of the pituitary gland for the diagnosis of Cushing's disease. Clin Endocrinol (Oxf) 49:293-300, 1998

46. Tsuchiya K, Mizutani Y, Hachiya J: Preliminary evaluation of fluid-attenuated inversion-recovery MR in the diagnosis of intracranial tumors. AJNR Am J Neuroradiol 17:10811086, 1996

47. Viacava P, Gasperi M, Acerbi G, Manetti L, Cecconi E, Bonadio AG, et al: Microvascular density and vascular endothelial grow th factor expression in normal pituitary tissue and pituitary adenomas. J Endocrinol Invest 26:23-28, 2003

48. Vitale G, Tortora F, Baldelli R, Cocchiara F, Paragliola RM, Sbardella E, et al: Pituitary magnetic resonance imaging in Cushing's disease. Endocrine 55:691-696, 2017

49. Wind JJ, Lonser RR, Nieman LK, DeVroom HL, Chang R, Oldfield EH: The lateralization accuracy of inferior petrosal sinus sampling in 501 patients with Cushing's disease. J Clin Endocrinol Metab 98:2285-2293, 2013

\section{Disclosures}

The authors report no conflict of interest concerning the materials or methods used in this study or the findings specified in this paper.

\section{Author Contributions}

Conception and design: all authors. Acquisition of data: Chittiboina, Chatain, Patronas, Smirniotopoulos, Piazza, Benzo, RayChaudhury, Sharma, Lodish, Nieman. Analysis and interpretation of data: all authors. Drafting the article: all authors. Critically revising the article: all authors. Reviewed submitted version of manuscript: all authors. Approved the final version of the manuscript on behalf of all authors: Chittiboina. Statistical analysis: all authors. Administrative/technical/material support: all authors. Study supervision: Chittiboina, Chatain, Patronas.

\section{Supplemental Information \\ Online-Only Content}

Supplemental material is available with the online version of the article.

Supplemental Table and Figure. https://thejns.org/doi/suppl/ 10.3171/2017.4.JNS17234.

\section{Correspondence}

Prashant Chittiboina, Neurosurgery Unit for Pituitary and Inheritable Diseases, National Institute of Neurological Diseases and Stroke, National Institutes of Health. 10 Center Dr., Rm. 3D20, Bethesda, MD 20892-1414. email: prashant.chittiboina@ nih.gov. 